\title{
Selection of the lasing frequency and heating the quantum-well heterostructure laser diodes
}

\author{
V. K. Kononenko ${ }^{1,2}$, B. F. Kuntsevich ${ }^{1}$ \\ ${ }^{1}$ Stepanov Institute of Physics NASB, Minsk, Belarus \\ ${ }^{2}$ Belarusian State University, Minsk, Belarus \\ vklavik@gmail.com
}

\begin{abstract}
Numerical simulation of controlling the lasing frequency for the $1.5 \mu \mathrm{m}$-range quantum-well heterostructure laser diodes is developed with taking into account increasing the active region temperature under pump current modulation. Peculiarities of the amplitude-frequency characteristics are analyzed at selection of the lasing frequency. General description of the heating of the laser active region during the current modulation is given and selection conditions of the lasing frequency that provide the response bandwidth up to $40 \mathrm{GHz}$ are established.
\end{abstract}

To solve some scientific and practical tasks there are required dynamic single-frequency semiconductor lasers with narrow and stable radiation line [1,2]. Besides, in a number of cases it is necessary to tune the lasing radiation frequency within the gain band. In particular, an important interest is a systematic research of output parameters of semiconductor lasers with a high-frequency pump current modulation and selection of the lasing frequency, which is used in fiber-optical information systems [3, 4]. One from the main problems of fiber-optical communication with wavelength-division multiplexing is development of laser emitters with line of width no more than $0.1 \mathrm{~nm}$ and maintaining the modulation signals with frequencies up to $10 \mathrm{GHz}$. To such a requirement there are the most suitable semiconductor lasers with an external Bragg fiber grating on single-mode waveguides [1].

In the work, using numerical modeling the amplitudefrequency characteristics and resonance properties of the response of semiconductor lasers emitting in the diapason of $1.5 \mu \mathrm{m}$ are investigated. These emitters based on the quantumwell (QW) GaInAs-GaInAsP heterosystem are the principal sources for fiber-optical communication networks. As shown, at typical laser diode parameters due to spectral selectivity it is possible in the range of $1.5 \mu \mathrm{m}$ to extend and get the modulation response bandwidth up to $\approx 40 \mathrm{GHz}$ [4].

At first, discuss a problem of change in the active region temperature under pump current modulation. By definition, a heating of the active region is equal to

$$
\Delta T=R_{T} s W_{T},
$$

where $R_{T}$ is the thermal resistance, $s$ is the contact square, $W_{T}$ is the dissipated thermal energy per unity square of $p-n$ junction. In general, we have (in $\mathrm{W} / \mathrm{cm}^{2}$ )

$$
W_{T}=R_{0} j^{2}+\frac{\eta^{\prime} h v_{\text {st }}}{e}((1-F) j+F f(j)) \text {. }
$$

Here, $R_{0} j^{2}$ determines physical irremovable cause of heating, i. e., Joule heating, the function $f(j)$ characterizes conditions of pumping the semiconductor source active region and

$$
f(j)=j_{\text {th }}+\gamma\left(j-j_{\text {th }}\right)=j_{\text {th }}+\left(1-\frac{\eta_{\mathrm{st}}}{\eta^{\prime}}\right)\left(j-j_{\text {th }}\right) .
$$

A threshold current density is $j_{\mathrm{th}}$, the parameter $\gamma=1-\eta_{\mathrm{st}} / \eta$ ' shows the part of the pump current above the threshold that is uselessly spend, $\eta_{\mathrm{st}}$ is the internal quantum yield of lasing, $\eta$ ' is the injection efficiency. Output function of a laser with the Fabry-Pérot type cavity equals $F=k_{\mathrm{r}} / k_{1}=k_{\mathrm{r}} /\left(k_{1}+\rho\right)$, where $k_{\mathrm{r}}=(1 / 2 l) \ln \left(1 / r_{1} r_{2}\right)$ is the useful output losses, $k_{1}=k_{\mathrm{r}}+\rho$ is the cavity loss coefficient, $\rho$ is the internal optical losses, $l$ is the cavity length, $r_{1}$ and $r_{2}$ are end reflectance.

Substituting (2) and (3) into (1), we obtain [5]

$$
\Delta T=R_{T} S\left(R_{0} j^{2}+\frac{\eta^{\prime} h v_{\mathrm{st}}}{e}\left(\left(1-\frac{\eta_{\mathrm{st}}}{\eta^{\prime}} F\right) j+\frac{\eta_{\mathrm{st}}}{\eta^{\prime}} F j_{\mathrm{th}}\right)\right) .
$$

Expression (4) has general character and it takes into account practically all the most important mechanisms and processes resulting in heating the active region of an injection laser or light-emitting diode. For a light-emitting diode we have $\eta_{\mathrm{st}}=0$, $F \approx 0, h v_{\mathrm{st}} \rightarrow h v \approx E_{\mathrm{g}}$ and therefore

$$
\Delta T=R_{T} s j\left(R_{0} j+\frac{\eta^{\prime} h v}{e}\right) .
$$

For a laser at the threshold $\left(j=j_{\text {th }}\right)$ we have

$$
\Delta T=R_{T} s j_{\text {th }}\left(R_{0} j_{\text {th }}+\frac{\eta^{\prime} h v_{\text {st }}}{e}\right) .
$$

In the general case, it has to account a dependence of the threshold on the active region temperature. In pulse regime one has to consider the threshold to be constant and for determining the laser active region temperature it is possible to use expression (4), where the ratio $j / j_{\mathrm{th}}=j_{\mathrm{b}} / j_{\mathrm{th}}$ is the threshold number (ratio of the direct current component to the threshold).

Typical values of the thermal resistance are $R_{T}=30 \div 120 \mathrm{~K} / \mathrm{W}$. To evaluate Joule heating let us use a value of the specific resistance of $R_{0}=R_{\mathrm{S}} \mathrm{S}=10^{-4} \Omega \mathrm{cm}^{2}$, where $R_{\mathrm{S}}$ is the series electrical resistance of the diode (at $s=5 \times 10^{-5} \mathrm{~cm}^{2}$, $R_{\mathrm{S}}=2 \Omega$ ). The thermal resistance value is assumed to be of $R_{T}=40 \mathrm{~K} / \mathrm{W}$. Mention ones more that thermal task is rather complex problem and it requires accounting a concrete structure of the laser device and conditions of pumping and of radiation output. 
For determining, calculations are developed for dynamical single-mode QW heterolasers in the GaInAs-GaInAsP system having in the active region two QWs of width each $\approx 5 \mathrm{~nm}$. It is considered that lasing occurs at a single longitudinal mode with the frequency $v_{\text {st }}$, which can to displace at any point of the gain band. To describe operation of the laser, use the rate equation system for the photon density $S$ in the cavity and for the nonequilibrium current carrier concentration $N$, similarly to [4], i.e.,

$$
\begin{gathered}
\dot{S}=v \mu\left(\Gamma k\left(v_{\mathrm{st}}\right)-k_{\mathrm{l}}\right) S+\beta N_{\mathrm{a}} R_{\mathrm{sp}}, \\
\dot{N}=\eta^{\prime} j / e d N_{\mathrm{a}}-R_{\mathrm{sp}} / \eta_{\mathrm{sp}}-v \mu \Gamma k\left(v_{\mathrm{st}}\right) S / N_{\mathrm{a}},
\end{gathered}
$$

where $v$ is the light velocity in the active region, $\mu$ is the coefficient of fulfillment of the active medium by cavity, $\Gamma$ is the confinement factor, $k\left(v_{\mathrm{st}}\right)$ and $k_{1}$ are coefficients of gain and losses, $R_{\mathrm{sp}}$ is the rate of spontaneous radiative recombination, $\beta$ is the coefficient determining the contribution part of spontaneous radiation into lasing mode, $j$ is the pump current density, $d$ is the thickness of the active layer (QW width), $N_{\mathrm{a}}$ is the number of QWs in the active region, $\eta^{\prime}$ is the injection efficiency, $\eta_{\mathrm{sp}}$ is the quantum yield of luminescence. The gain spectrum $k\left(v_{\mathrm{st}}\right)$ and rate of spontaneous radiative recombination $R_{\text {sp }}$ are determined in the framework of the optical model of the active medium with no the $\mathbf{k}$-selection rule on the electron wave vector for ground transitions [3, 4].

Modulation of pump current is described in the form, as $j=j_{\mathrm{b}}+j_{\mathrm{m}} \sin \left(2 \pi v_{\mathrm{m}} t\right)$, where $j_{\mathrm{m}}$ and $v_{\mathrm{m}}$ are depth and frequency of modulation, and $j_{\mathrm{b}}$ is the direct current constant component. It is defined that $j_{\mathrm{b}}=x_{\mathrm{b}} j_{\mathrm{th}}$ and $j_{\mathrm{m}}=x_{\mathrm{m}} j_{\mathrm{b}}$, where $x_{\mathrm{b}}$ and $x_{\mathrm{m}}$ are variable parameters, $j_{\text {th }}=e d N_{\mathrm{a}} R_{\mathrm{sp}-\mathrm{th}} / \eta^{\prime} \eta_{\mathrm{sp}}$ is the stationary threshold, $R_{\text {sp-th }}$ is the threshold rate of spontaneous recombination.

At calculations, there are determined for the GaInAsGaInAsP system the following parameter values, i.e., $d=5 \mathrm{~nm}, \quad N_{\mathrm{a}}=2, \quad \Gamma=0.845 \times 10^{-2}, \quad \beta N_{\mathrm{a}}=10^{-5}, \quad k_{1}=25 \mathrm{~cm}^{-1}$, $\mu=1, \eta_{\mathrm{st}} F / \eta^{\prime} \approx 0.6$. Values of the effective masses $m_{\mathrm{c}}, m_{\mathrm{vh}}$, $m_{\mathrm{vl}}, m_{\mathrm{vht}}$, and $m_{\mathrm{vlt}}$, of parameters of heterostructure components and, respectively, of the subband levels $E_{\mathrm{c} 1}, E_{\mathrm{vh} 1}$, and $E_{\mathrm{vl} 1}$ are found based on some published data (e.g., [2]). In particular, we determine initial quantities, as $m_{\mathrm{c}} / m_{\mathrm{e}}=0.041$, $m_{\mathrm{vh}} / m_{\mathrm{e}}=0.31, \quad m_{\mathrm{vl}} / m_{\mathrm{e}}=0.040, \quad m_{\mathrm{vht}} / m_{\mathrm{e}}=0.052, \quad$ and $m_{\mathrm{vlt}} / m_{\mathrm{e}}=0.12$. Then, we obtain $E_{\mathrm{c} 1}=69.7 \mathrm{meV}$, $E_{\mathrm{vh} 1}=27.0 \mathrm{meV}$, and $E_{\mathrm{v} 11}=88.4 \mathrm{meV}$, and the initial transition photon energy is $h v_{1}=E_{\mathrm{g}}+E_{\mathrm{c} 1}+E_{\mathrm{vh} 1}=815 \mathrm{meV}\left(\lambda_{1}=1.5 \mu \mathrm{m}\right)$, where the energy gap at room temperature is $E_{\mathrm{g}}=718 \mathrm{meV}$. In the temperature interval from 300 to $400 \mathrm{~K}$ we have approximately (in eV) $E_{\mathrm{g}}(T)=0.718-3.776 \times 10^{-4}(T-300)$. Therewith, the probability of optical transitions follows a law $A(T)=A_{0}-4.454 \times 10^{-6}(T-300)$, where at room temperature $A_{0}=4.35 \times 10^{-3} \mathrm{~cm}^{2} / \mathrm{s}$.

The threshold data are determined as follows. By means of a selection we find such a quasi-Fermi level difference $\Delta F_{\text {th }}$ that at a some point $v_{\mathrm{st}}$ of the gain band the certain condition for the maximum gain coefficient with no lasing is fulfilled, i.e., $\Gamma k\left(v_{\mathrm{st}}\right)=g_{\mathrm{hm}}\left(v_{\mathrm{st}}\right)=k_{\mathrm{l}}$ (see Fig. 1). After calculations of $R_{\mathrm{sp}-\mathrm{th}}$, the threshold current density is received. For the studied system, near the gain spectrum center we obtain (at room temperature) $\quad h v_{\text {th }}=831 \mathrm{meV}, \quad \Delta F_{\text {th }}=848 \mathrm{meV}, \quad$ and $j_{\mathrm{th}} \approx 494 \mathrm{~A} / \mathrm{cm}^{2}\left(\right.$ at $\eta^{\prime} \approx 1$ and $\left.\eta_{\mathrm{sp}} \approx 1\right)$.

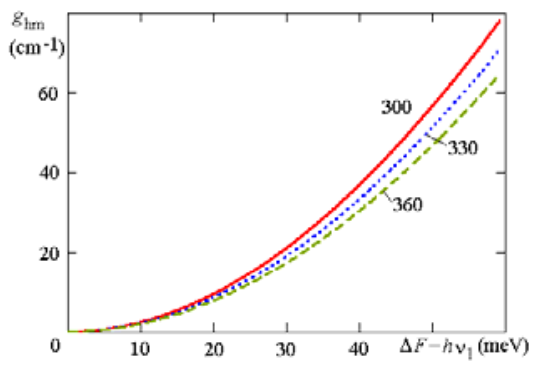

Fig. 1. Dependence of the maximum modal gain $g_{\mathrm{hm}}$ on the quasi-Fermi level difference $\Delta F$ in the active region. Numbers at the curves are operation temperature (K).

When selecting the lasing wavelength within the gain band the threshold quasi-Fermi level difference $\Delta F_{\text {th }}$ in the active region follows a specific law (Fig. 2). Results for $j_{\text {th }}$ obtained in Fig. 3 were predicted earlier in [6] and demonstrated for the selectively tunable GaSb-based QW laser diodes in [7].

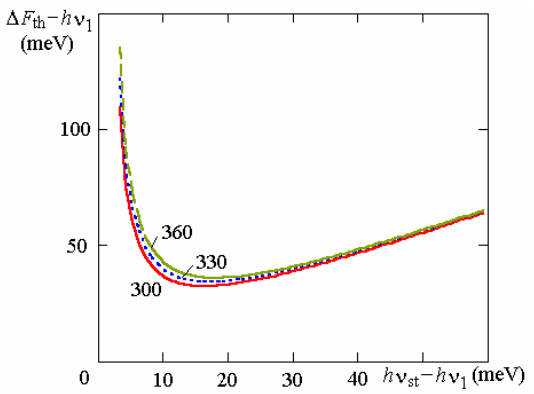

Fig. 2. Dependence of the threshold quasi-Fermi level difference $\Delta F_{\text {th }}$ in the active region on the displacement of lasing frequency $v_{\text {st }}$ within the gain band. Numbers at the curves are operation temperature $(\mathrm{K})$.

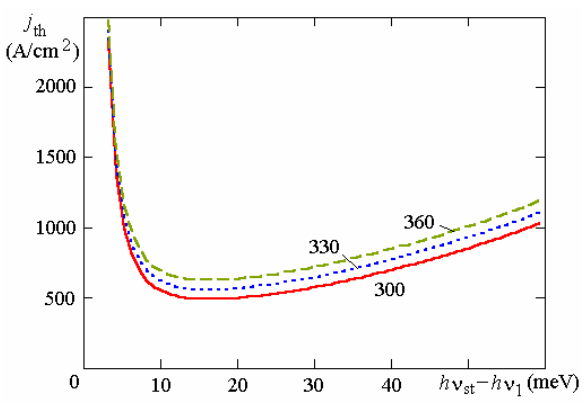

Fig. 3. Change in the density of threshold current $j_{\text {th }}$ versus selection of lasing frequency $v_{\mathrm{st}}$ within the gain band. Numbers at the curves are operation temperature (K).

Numerical calculations versus operation temperature are produced using the following obtained expressions for quantities of electron and hole chemical potentials, i.e, 


$$
\begin{aligned}
\varsigma_{\mathrm{c}}(\Delta, T) & =79.227+7.626 \times 10^{-2}(T-300) \\
+ & \left(0.584+0.013 \times 10^{-2}(T-300)\right)\left(\Delta-\Delta_{1}\right), \\
\varsigma_{\mathrm{h}}(\Delta, T) & =17.473-7.626 \times 10^{-2}(T-300) \\
& +\left(0.416-0.013 \times 10^{-2}(T-300)\right)\left(\Delta-\Delta_{1}\right) .
\end{aligned}
$$

Obviously, the sum $\zeta_{\mathrm{c}}+\zeta_{\mathrm{h}}$ is equal to $\Delta$, where $\Delta_{1}=96.7 \mathrm{meV}$ corresponds to starting the transitions between the first (ground) electron subband and ground heavy hole subband and $\Delta_{2}=158.1 \mathrm{meV}$ corresponds to starting the transitions between the electron and light hole subbands

General results of the numerical simulation show that changes in direct pump current component, modulation frequency, and in displacement of lasing line within the gain band exert comparatively strong influence on temporal and energy parameters of emission in QW heterolasers. As seen from Fig. 4, if selection occurs at the long-wavelength edge of the gain band $\left(h v_{\mathrm{st}}<0.83 \mathrm{eV}\right)$, the heating at the number of threshold of the order of $8-10$ reaches up to $12 \mathrm{~K}$. Therewith, the maximum gain coefficient moves (from $1.49 \mu \mathrm{m}$ to $1.52 \mu \mathrm{m})$ towards the lasing wavelength and response characteristics become more improved. The heating at pump current modulation in the case of initial selection of the lasing frequency near the center of the gain band deteriorates the response characteristics because the lasing wavelength deviates from optimal conditions. The worse conditions occur at the short wavelength edge of the gain band $\left(h v_{\mathrm{st}}>0.83 \mathrm{eV}\right)$, where the heating can be also of $10 \mathrm{~K}$ but the maximum gain coefficient is more shifted, as compared the lasing wavelength, and therefore response characteristics deteriorate.

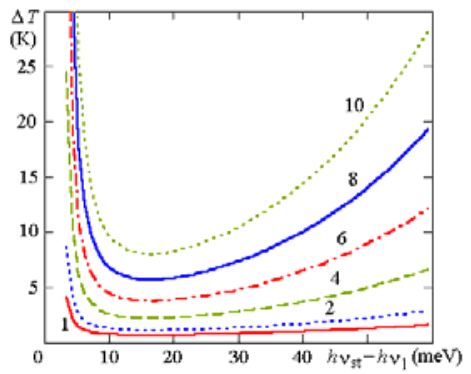

Fig. 4. Growth of the active region temperature $\Delta T$ versus selection of the lasing frequency $v_{\text {st }}$ within the band gain and the number of threshold (figures at the curves). Curve 1 is related to initial conditions at room temperature.

Maximum values of the response bandwidth $v_{-3 \mathrm{~dB}}$ occur at the central part of the gain band. Therewith, increase of the direct current component influences due to heating on quantity of $v_{-3 \mathrm{~dB}}$ and results in shifting the corresponding $v_{\mathrm{st}}$ into the high-frequency region. At the used system parameters the maximum value $v_{-3 \mathrm{~dB}}$ reaches of $\approx 40 \mathrm{GHz}$ (Fig. 5). The laser amplitude-frequency characteristics at definite parameters display two local maxima. Low-frequency maximum corresponds ordinary to sub-harmonic $1 / 2$, and high-frequency one to the main resonance [4]. Mention that at a powerful excitation, where heating is essential, the modulation depth of output radiation changes versus the modulation frequency $v_{\mathrm{m}}$.
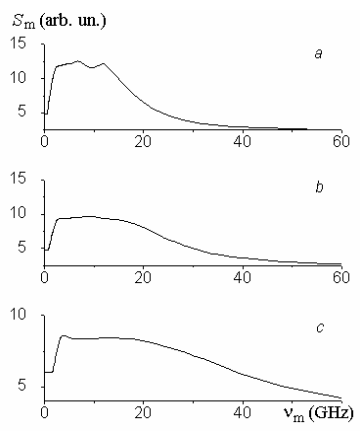

Fig. 5. Amplitude-frequency characteristics $S_{\mathrm{m}}\left(v_{\mathrm{m}}\right)$ of a laser, (a) $h v_{\mathrm{st}}=824$, (b) 831, and (c) $844 \mathrm{meV}, x_{\mathrm{b}}=10, x_{\mathrm{m}}=1$.

In conclusion, influence of tuning the lasing frequency and of heating on the response of the QW GaInAs-GaInAsP laser diodes is examined in detail. Established features are applied at development of effective optical information systems.

\section{REFERENCES}

[1] V. P. Duraev, E. T. Nedelin, T. P. Nedobyvailo, M. A. Sumarokov, and K. I. Klimov, "Bragg fibre grating semiconductor lasers with the narrow emission spectrum in the $1530-1560-\mathrm{nm}$ region," Quantum Electron., 2001, vol. 31, no.6, pp. 529530.

[2] B. F. Kuntsevich, V. K. Kononenko, and A. N. Pisarchik, "Controlling dynamics in parametrically modulated lasers with cavity detuning," in Recent Advances in Laser Dynamics: Control and Synchronization, Research Signpost, Kerala, India, 2008, ch. 5, pp. 221-267.

[3] V. K. Kononenko, B. F. Kuntsevich, and M. Marciniak, "Nonlinear dynamics of directly modulated quantum-well heterostructure laser diodes and deviation of the emission frequency," Nonlinear Phenomena in Complex Systems, 2008, vol. 11, no. 4, pp. 429-439.

[4] B. F. Kuntsevich and V. K. Kononenko, "Modulation response of dynamic single-mode quantum-well heterolasers in the $1.5 \mu \mathrm{m}$ range," J. Appl. Spectrosc., 2010, vol. 77, no. 4, pp. 541-548.

[5] V. K. Kononenko and G. T. Pak, "Heating the laser diodes," Techn. Phys. Lett., 1982, vol. 8, no. 12, pp. $750-754$.

[6] A. G. Buikevich, I. S. Manak, and V. K. Kononenko, "Polarization of the radiation of a size-quantized heterojunction laser with a selective cavity," J. Opt. Technol., 2004, vol. 71, no. 11, pp. 734-736.

[7] E. Geerlings, M. Rattunde, J. Schmitz, G. Kaufel, H. Zappe, and J. Wagner, "Widely tunable GaSbbased external cavity diode laser emitting around 2.3 um," IEEE Photon. Technol. Lett., 2006, vol. 18, no. 18 , pp. 1913-1915. 